\title{
AEROBIOLOGÍA Y BIODETERIORO DEL GÉNERO Aspergillus Link EN DEPÓSITOS DE TRES INSTITUCIONES PATRIMONIALES CUBANAS
}

\section{(AEROBIOLOGY AND BIODETERIORATION OF THE GENUS Aspergillus Link IN REPOSITORIES OF THREE CUBAN HERITAGE INTITUTIONS)}

\author{
Alian Molina V., Sofía Borrego A. \\ Laboratorio de Conservación Preventiva, Archivo Nacional de la República de Cuba, \\ Compostela 906 esquina a Calle San Isidro, La Habana Vieja, La Habana, Cuba. \\ Contactos: alian@arnac.cu,sofia@arnac.cu \\ RECIBIDO:2 de Marzo de 2016 \\ APTOBADO:3 de Mayo de 2016
}

LOS AUTORES DECLARAN NO TENER CONFLICTO DE INTERESES

Palabras clave: Aspergillus; biodeterioro; estudios aerobiológicos; patrimonio cultural; patógeno oportunista.

Keywords: Aspergillus; aerobiological studies; biodeterioration; cultural heritage; opportunistic pathogen.

\section{RESUMEN}

Las especies del género Aspergillus Link constituyen importantes agentes biodeteriorantes de objetos de valor constructivo, cultural y patrimonial. En ambientes laborales, sus propágulos y metabolitos pueden provocar afecciones a la salud humana. Los estudios aeromicológicos pueden contribuir a minimizar el impacto de Aspergillus spp. en el biodeterioro y la calidad de vida. Se han caracterizado de acuerdo a su potencial biodeteriógeno y patogénico representantes de Aspergillus spp. en el aire de depósitos de materiales en tres instituciones patrimoniales cubanas. Las muestras se tomaron empleando un biocolector SAS súper 100. Se evaluó cualitativamente la actividad celulolítica, amilolítica y proteolítica de los aislados; así como la excreción de pigmentos y ácidos or- gánicos. Se evaluó el crecimiento a $37^{\circ} \mathrm{C}$ y la secreción de hemolisinas. Aspergillus spp. constituyó parte considerable de la micobiota aérea viable en los depósitos con 22 especies correspondientes a 12 secciones, las de mayor variabilidad fueron Flavi y Restricti, mientras la primera y la sección Aspergillus fueron las mejor representadas. El 74\% de las cepas mostraron alta capacidad para degradar los compuestos orgánicos evaluados y secretar ácidos, mientras el 34\% excretó pigmentos de amarillo a pardo oscuro, se evidenció un amplio potencial biodeteriorante en general. Más del 30\% de los aislados mostraron ser patógenos oportunistas peligrosos para el hombre al crecer a $37^{\circ} \mathrm{C}$, secretar hemolisinas, y presentar un tamaño conidial que posibilita la depositación en alvéolos pulmonares. Las cepas con mayor impacto biodeteriorante y patogénico fueron las correspondientes a 
las Secciones Flavi, Nigri y la especie Aspergillus fumigatus Fresen.

\section{ABSTRACT}

Species of the genus Aspergillus Link cause damage to objects constructive, cultural and heritage value. In work environments, their propagules and metabolites may cause disorders to human health. Aerobiological studies can help minimize the impact of Aspergillus spp. in the biodeterioration and quality of life. Aspergillus spp. strains present in the air of the repositories in three Cuban heritage institutions were characterized according to their biodeteriogenic and pathogenic potential. Each isolated was classified taxonomically, cellulolytic, amylolytic and proteolytic activity as well as excretion of pigments and organic acids was evaluated. The capacity to grow at $37^{\circ} \mathrm{C}$ and secrete hemolysins was qualitatively evaluated. Aspergillus spp. was significant portion of viable air micobiota in the three repositories with 22 species corresponding to 12 sections, most variability were Flavi and Restricti, while the first section and $A s-$ pergillus were the best represented. The $74 \%$ of the strains showed high ability to degrade organic compounds evaluated and secrete acids, while 34\% excreted pigments from yellow to dark brown, evidenced a broad biodeteriogenic potential in general. More than $30 \%$ of the isolates were shown to be dangerous opportunistic pathogens for humans to grow at $37^{\circ} \mathrm{C}$, secrete hemolysin, and present a conidial size which enables the deposition pulmonary alveoli. Strains higher biodeteriogenic and pathogenic impact were those corresponding to the Flavi, Nigri sections and the species Aspergillus fumigatus Fresen.

\section{INTRODUCCIÓN}

El género Aspergillus Link, tiene gran importancia desde el punto de vista natural, social y económico. Sus especies están entre los hongos más abundantes del mundo. Pueden encontrarse en varios nichos ecológicos y repercuten de forma considerable en el ecosistema al ser descomponedores de la materia orgánica. (Piontelli, 2008; Krijgsheld et al., 2012). En procesos biotecnológicos como la clonación de determinados genes, la obtención de metabolitos, antibióticos, ácidos orgánicos y enzimas se utilizan frecuentemente cepas de Aspergillus, además de su uso como microorganismos industriales en diversos procesos fermentativos para la producción de alimento humano y animal (Samson et al., 2014). Dicho género se ha detectado frecuentemente en estudios aeromicológicos de ambientes interiores y exteriores de regiones tropicales del planeta como parte importante de la micobiota total aérea y mostrando gran variabilidad de grupos y especies (Rojas et al., 2007; Nevalainen y Morawska, 2009; Almaguer y Rojas et al., 2013). De forma general los representantes de Aspergillus spp. son colonizadores primarios de los sustratos, al estar respaldados por una potente y versátil maquinaria metabólica, una amplia capacidad xerófila $(a w \leq 0.90)$ y una eficiente forma de dispersión (anemocoría) a través de numerosos propágulos (Rojas et al., 2009; Ruth et al, 2012; Micheluz et al., 2015). Son capaces de colonizar una amplia variedad de sustratos; se pueden encontrar frecuentemente como contaminantes de alimentos, granos, semillas y conservas lo cual deriva en pérdidas económicas considerables (Sepúlveda y Piontelli, 2005; Anaya et al., 2014). Varios grupos y especies del género se han señalado como importantes agentes biodeteriorantes de la madera, los textiles, el papel e incluso polímeros sintéticos y materiales pétreos siendo la causa de serios daños a objetos de valor constructivo, cultural y patrimonial. (Ljaljević y Vukojević, 2009; Borrego et al., 2010; Bučková, et al., 2014; Rodríguez et al., 2014). En interiores las fuentes de esporas u otros propágulos de Aspergillus spp. pueden provocar afecciones a la salud, riesgo que aumenta en ambientes laborales (alta exposición por largos períodos de tiempo). En individuos inmunodeficientes representantes de algunas especies o grupos pueden producir desde reacciones alérgicas leves hasta enfermedades severas. Una de las más rele- 
vantes es la aspergillosis invasora con altos valores de morbilidad y mortalidad (Oliveira y Caramalho, 2014). Los estudios aeromicológicos en ambientes interiores de riesgo y la caracterización de las cepas predominantes, pueden contribuir a minimizar el impacto de Aspergillus spp. en el biodeterioro y la calidad de vida, sin embargo estos son escasos en Cuba. El objetivo fue caracterizar de acuerdo a su potencial biodeteriorante y patogénico los representantes del género Aspergillus presentes en el aire de depósitos de materiales en tres instituciones patrimoniales situadas en el municipio Habana Vieja, Cuba.

\section{MATERIALES Y MÉTODOS}

\section{Características de los depósitos}

El estudio se realizó en tres edificios de instituciones patrimoniales ubicadas en el Municipio Habana Vieja, La Habana, Cuba. En cada uno se escogió un depósito que resguarda colecciones de interés científico e histórico cultural. Las características de los depósitos y las colecciones se recogen en la tabla I.

\section{Muestreo y análisis micológico del aire}

Los muestreos se realizaron en febrero de 2014 (época de seca) entre las 11 y las 12 horas considerando el reporte de mayor concentración fúngica en el aire de la ciudad (Almaguer y Rojas, 2013). Los puntos de muestreo en cada local se seleccionaron de acuerdo con Sánchis (2002) y las muestras se tomaron por triplicado empleando un biocolector SAS Super 100 (Italia) a intervalos de 1 hora. Se tomaron $100 \mathrm{~L}$ de aire en un tiempo de 1 min. El medio de cultivo utilizado fue Agar Malta (AM) suplementado con $\mathrm{NaCl}$ (7.5\%) (Rojas, 2008). Posteriormente las placas se incubaron invertidas a $28^{\circ} \mathrm{C} \pm 1^{\circ} \mathrm{C}$ durante 7 días, se realizó el conteo para determinar la concentración fúngica expresada en Unidades Formadoras de Colonias por metro cúbico de aire (UFC.m-3) y se realizó el aislamiento y depuración de las diferentes colonias de Aspergillus spp.

La clasificación taxonómica de los representantes del género se realizó de acuerdo con Barnett y Hunter (2003). Para ello, se tuvo en cuenta las características culturales y morfológicas observadas en microcultivos, preparaciones en fresco y semipermanentes con lactofenol, según las técnicas de Casadesús y Rojas (1981). Las observaciones se realizaron en un microscopio de campo claro (Olympus, Japón). Para la identificación de especies se siguieron los procedimientos de Klich y Pitt (1994). Estas metodologías están basadas primeramente en los caracteres morfológicos y en características fisiológicas como son las relaciones agua - temperatura, la pigmentación y el grado de desarrollo de las colonias en ciertos medios. Estas características se determinaron a los 7 días de inoculadas las cepas en los medios Agar Czapek Extracto de Levadura, incubadas a $25^{\circ} \mathrm{C}$ y $37^{\circ} \mathrm{C}$, y en los medios Agar Extracto de Malta y Agar Czapek Extracto de Levadura con $20 \%$ Sacarosa incubadas a $25^{\circ}$ C. Se consideraron además, para la identificación de ciertas especies las metodologías y criterios taxonómicos de Raper y Fennel (1965), Piontelli (2008) (Sección Fumigati) y Simões et al. (2013) (Sección Nigri).

Tabla 1. Características de los depósitos y las colecciones que resguardan.

\begin{tabular}{lcccc}
\hline Depósito & Edificio/Ubicación & Tipo de Colección & Soporte & Ventilación \\
\hline ARNAC & Archivo Nacional de Cuba (primera planta) & Documentos & Papel y Cartulina & Natural \\
\hline OCPI & $\begin{array}{c}\text { Oficina Cubana de la Propiedad Industrial } \\
\text { (planta baja) }\end{array}$ & Documentos & Papel y Cartulina & Climatización \\
\hline \multirow{2}{*}{ MNBA } & Museo Nacional de Bellas Artes (sótano) & $\begin{array}{c}\text { Esculturas, Instalaciones } \\
\text { y Cuadros }\end{array}$ & Madera, Yeso y Textiles & Climatización \\
\hline
\end{tabular}




\section{Análisis Biométricos y estadísticos}

Se determinó la densidad relativa (DR) de cada taxa (Smith, 1980) donde: $\mathrm{DR}=$ (número de colonias de un taxa/número total de colonias) $\mathrm{x}$ 100. El análisis estadístico, realizado con el programa Statgraphics Centurion XV, incluyó una prueba de t-Student para analizar la distribución de probabilidades, determinar su normalidad y realizar las comparaciones entre las concentraciones fúngicas en los depósitos (ARNAC, OCPI y MNBA).

\section{Determinación de atributos biodeteriorantes}

\section{Determinación de la actividad proteolítica}

La actividad proteolítica cualitativa fue determinada a través de la hidrólisis de la gelatina en tubos de cultivo implementando una modificación al método de Abrusci et al. (2005) que fue referido por Borrego et al. (2010). Los tubos inoculados fueron incubados durante 7 días a $28^{\circ} \mathrm{C} \pm 1^{\circ} \mathrm{C}$ y posteriormente fueron incubados a $4^{\circ} \mathrm{C}$ durante 1 hora. Se evidenció la reacción de hidrólisis por la licuefacción del medio al agitar los tubos moderadamente.

\section{Determinación de la actividad celulolítica}

Con el objetivo de determinar el poder degradativo de tres tipos de fuentes de celulosa las cepas fúngicas se cultivaron en tubos de medio agarizado de composición salina según Borrego et al. (2010) y Lavín et al. (2014). Como fuente de carbono se empleó en un caso una tira de papel filtro (Whatman $\mathrm{n}^{\circ} 1$, Inglaterra), en los otros dos ensayos se emplearon al 1\% carboximetil celulosa y celulosa cristalina respectivamente. Como control se empleó glucosa (1\%). Los cultivos se incubaron a $28^{\circ} \mathrm{C} \pm 1^{\circ} \mathrm{C}$ durante 21 días.

\section{Producción de pigmentos}

Se determinó por observación en la misma tira de papel de filtro (Whatman $n^{\circ} 1$, Inglaterra), utilizada en la prueba descrita anteriormente.

\section{Determinación de la actividad amilolítica}

La actividad amilolítica se determinó a través de la hidrólisis del almidón en placas, se utilizó un medio de composición salina similar al empleado por Borrego et al. (2010) y como fuente de car- bono almidón (1\%). Después de incubar durante 7 días a $28^{\circ} \mathrm{C} \pm 1^{\circ} \mathrm{C}$, se vertieron sobre cada placa de cultivo $5 \mathrm{ml}$ de solución del reactivo Lugol. Una zona incolora alrededor de la colonia fue tomada como indicador de hidrólisis positiva (Guiamet et al., 2011).

5. Determinación cuantitativa de la producción de ácidos orgánicos

Se realizaron suspensiones de esporas de la cepas y se sembraron en un caldo de cultivo de composición salina según indica Borrego et al. (2010). Como única fuente de carbono se empleó glucosa (1\%). Los cultivos se incubaron a $28^{\circ} \mathrm{C} \pm 1^{\circ} \mathrm{C}$ durante 3 días y posteriormente se midió el $\mathrm{pH}$ del medio de cultivo con la ayuda de un potenciómetro (Pacitronic MV 870, USA).

\section{Determinación de atributos patogénicos}

\section{Crecimiento a $37^{\circ} \mathrm{C}$}

Las cepas fueron sembradas en placas que contenían como medio de cultivo Agar Malta (AM). Se incubaron durante 7 días a $37^{\circ} \mathrm{C} \pm 1^{\circ} \mathrm{C}$, para observar si las cepas evaluadas crecían a esa temperatura (Llop et al., 2001).

\section{Producción de hemolisinas}

Las cepas fueron sembradas en placas Petri que contenían como medio de cultivo Agar Czapeck (ACZ), al cual se le agregó $5 \mathrm{ml}$ de sangre de carnero desfibrinada por $95 \mathrm{ml}$ de medio. Posteriormente se inocularon e incubaron a $28^{\circ} \mathrm{C} \pm 1^{\circ} \mathrm{C}$ durante 7-10 días. La actividad hemolítica se evidenció por la aparición de un halo verdoso o transparente alrededor de la colonia en el medio rojo (Bogomolova y Kirtsideli, 2009).

En todos los casos los experimentos anteriores se realizaron por triplicado con controles positivos y negativos.

\section{RESULTADOS Y DISCUSION}

\section{Análisis Ecológico}

Los representantes de Aspergillus spp. en los tres depósitos constituyeron una parte considerable de la micobiota aérea viable si se tienen en 
cuenta que las DR fueron superiores al 18\%, que en todos los locales se detectaron al menos 6 géneros fúngicos y que en términos de concentración, se ubicó siempre entre los tres géneros más representados con un máximo de $45 \mathrm{UFC}^{-3}$ en MNBA (figura 1A). Resultados similares fueron obtenidos en interiores por Codina et al. (2008) (54 casas de Florida, USA), Rojas y Aira (2012), Rodríguez et al. (2014), Borrego y Molina (2014) y Bipasha et al., 2015 (depósitos de archivos y museos). El análisis de las variables ambientales evidenció una correlación fuerte positiva entre la $\mathrm{HR}(\mathrm{R}=0,7449$ para $\mathrm{P} \leq 0.05)$ y las concentraciones del género Aspergillus en todos los casos, cuestión que está relacionada con la influencia de dicho factor en la presencia y viabilidad de sus propágulos (Rodríguez et al., 2014). En tanto la T y las concentraciones de propágulos mostraron una correlación baja negativa $(\mathrm{R}=-0,4878$ para $\mathrm{P} \leq 0.05$ ) (figura $1 \mathrm{~B}$, C y D). La T suele ser influyente en la dinámica de la micobiota en el aire interior, sin embargo al parecer ejerce un efecto secundario al actuar sobre otros factores como la HR y las corrientes de aire (Rodríguez et al., 2014). Por otra parte, se ha reportado la prevalencia de especies de Aspergillus en ambientes interiores con alto nivel de empolvamiento y falta de ventilación, elementos que propician la existencia de reservorios esporales (Molina
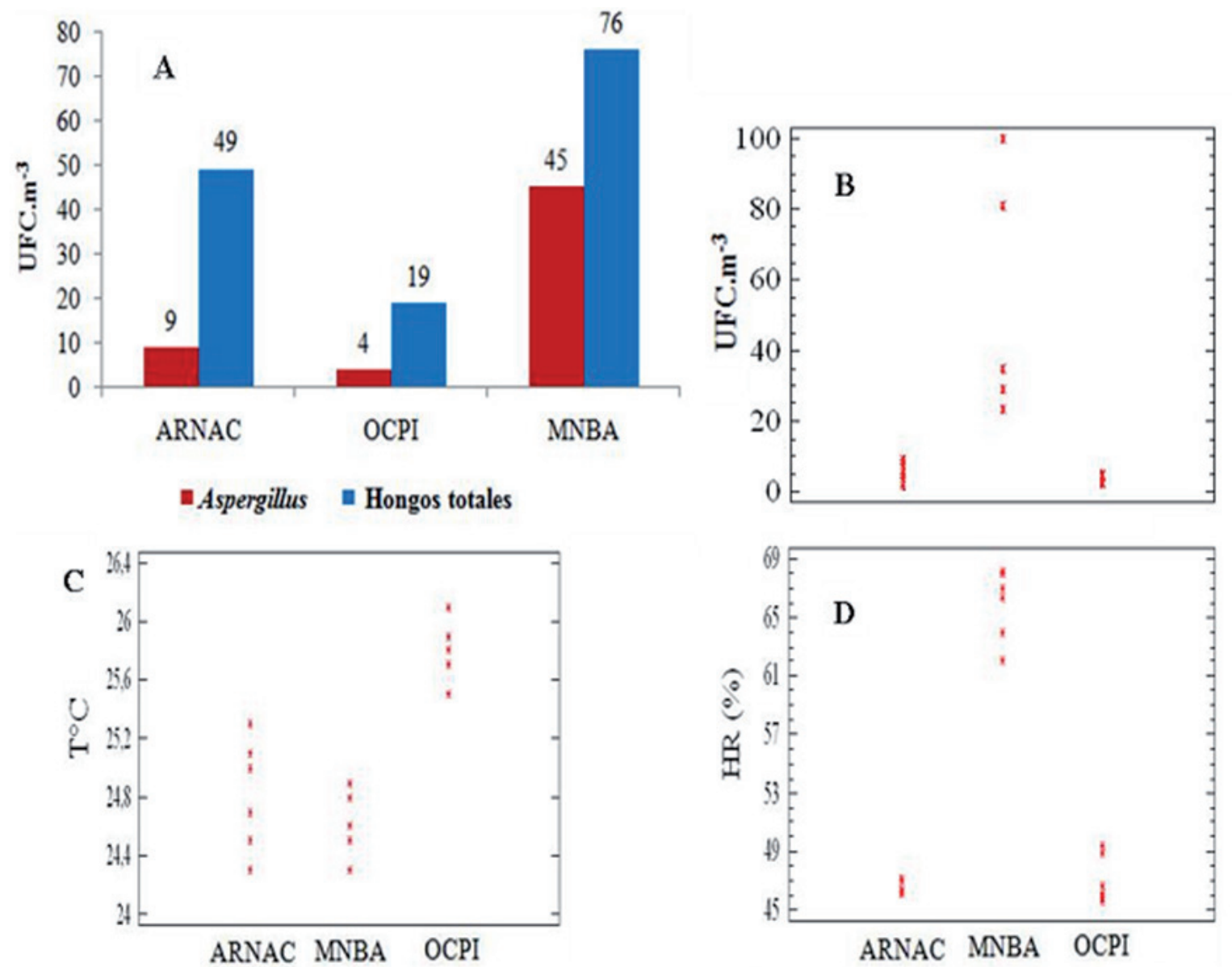

Figura 1. A: Valores promedio en $\mathrm{UFC}^{-3}$ de Aspergillus spp. con respecto a la micobiota aérea total viable en cada depósito. B, C y D: Gráficas de dispersión de datos (UFC ${ }^{-3}$ de Aspergillus spp., T y HR), obtenidas a partir del Análisis de Varianza (ANOVA simple). Nótese la similitud entre las gráficas B y D producto de la correlación fuerte positiva $(\mathrm{R}=0,7449$ para $\mathrm{p} \leq 0.05)$ entre la HR y las $\mathrm{UFC}^{-3}$. 
y Borrego, 2014; Micheluz et al., 2015). Horner et al. (2004) destacan que el predominio de hongos del suelo en un local, suele ser indicativo de colonización fúngica de superficies, presencia de agua o problemas de humedad. Lo anterior se corroboró en MNBA donde los valores de HR fueron superiores al $65 \%$ máximo que se recomienda para la conservación en archivos y museos cubanos (Resolución No. 41, 2009), se detectó crecimiento micelial sobre piezas de yeso, madera y tela además de un alto grado de empolvamiento. En ARNAC y OCPI donde las concentraciones fueron significativamente menores y no difirieron entre sí, la HR fue más baja y no se observó crecimiento fúngico activo. Como se ha sugerido, el género bajo determinadas circunstancias puede considerarse un bioindicador de la calidad ambiental de interiores, si se realiza además una buena identificación taxonómica (Cabral, 2010; Gaikwad y Sonawane, 2012).
Los 38 aislados se ubicaron en 22 especies correspondientes a 12 secciones diferentes, las de mayor variabilidad fueron Flavi y Restricti con 4 y 3 especies respectivamente (tabla II). La primera, conjuntamente con la sección Aspergillus fueron las 2 mejor representadas con al menos una especie en cada uno de los depósitos. En concordancia con este estudio, representantes de la sección Flavi se han reportado como contaminantes muy comunes del aire en bibliotecas, museos y almacenes en la Habana, Cuba. Han sobresalido las especies Aspergillus flavus Link y A. oryzae (Ahlburg) Cohn. por sus altas densidades relativas, en tanto también se ha detectado $A$. parasiticus Speare (Rojas y Aira, 2012). Sterflinger (2010) reportó A. flavus como uno de los hyphomycetes más frecuentes en museos y depósitos de obras de arte. Varios integrantes del grupo se detectaron en ambientes interiores de diferentes características en varias regiones del

Tabla II. Densidades relativas de los taxa identificados en cada uno de los depósitos analizados.

\begin{tabular}{|c|c|c|c|c|}
\hline \multicolumn{2}{|l|}{ Identificación taxonómica } & \multirow{2}{*}{\multicolumn{3}{|c|}{$\begin{array}{c}\text { Densidades relativas con respecto a la } \\
\text { micobiota total viable (\%) }\end{array}$}} \\
\hline \multirow{2}{*}{ TAXA } & \multirow{2}{*}{ SECCIÓN } & & & \\
\hline & & ARNAC & OCPI & MNBA \\
\hline A. chevalieri L. Mangin * & \multirow{2}{*}{ Aspergillus } & 0 & 0.9 & 2.53 \\
\hline A. glaucus Link (grupo) & & 5.55 & 0 & 29.11 \\
\hline A. candidus Link & Candidi & 0 & 2.72 & 0 \\
\hline A. clavatofiavus Raper \& Fennel & \multirow{4}{*}{ Flavi } & 2.7 & 0 & 0 \\
\hline A. flavus Link & & 0 & 1.81 & 1.26 \\
\hline A. oryzae (Ahlburg) Cohn. & & 0 & 0.9 & 0 \\
\hline A. parasiticus Speare & & 1.38 & 0 & 0 \\
\hline A. flavipes (Bainier \& Sartory) Thom \& Church & \multirow{2}{*}{ Flavipedes } & 0 & 0.9 & 1.26 \\
\hline A. niveus Blochwitz & & 0 & 0.9 & 6.32 \\
\hline A. fumigatus Fresen. & Fumigati & 0 & 0 & 1.26 \\
\hline A. unguis (Emile-Weill \& L. Gaudin) Thom \& Raper & Nidulantes & 0 & 5.45 & 0 \\
\hline A. niger van Tieghem & \multirow{2}{*}{ Nigri } & 1.38 & 1.81 & 0 \\
\hline A. tubingensis Mosseray & & 1.38 & 0 & 0 \\
\hline A. ochraceus K. Wil. & Ochraceoreosei & 0 & 0 & 1.26 \\
\hline A. conicus Blochwitz & \multirow{3}{*}{ Restricti } & 1.38 & 0 & 0 \\
\hline A. proliferans G. Smith & & 0 & 0 & 1.26 \\
\hline A. restrictus $\mathrm{G}$. Smith & & 4.1 & 0 & 2.53 \\
\hline A. conjunctus Kwon-Chung \& Fennel & \multirow{2}{*}{ Usti } & 0 & 0 & 1.26 \\
\hline A. ustus (Bainier) Thom \& Church & & 0 & 0 & 6.32 \\
\hline A. janus Raper \& Thom & \multirow{2}{*}{ Versicolor } & 0 & 0 & 1.26 \\
\hline A. versicolor (Vuill.) Tiraboschi & & 0 & 0.9 & 2.53 \\
\hline A. wentii Wehmer & Wentii & 0 & 0.9 & 0 \\
\hline
\end{tabular}


mundo (Nevalainen y Morawska, 2009). Las especies correspondientes al grupo Flavi se adaptan a diferentes condiciones medioambientales y son capaces de crecer a expensa de una amplia variedad de sustratos cuestión que condiciona su prevalencia en disimiles ambientes interiores. Estas, al igual que representantes del grupo Niger (detectados en este estudio) se han categorizado como xerotolerantes y termotolerantes pudiendo desarrollarse en un amplio rango de temperaturas y actividades de agua (Krijgsheld et al., 2012). Lo anterior pudiera explicar su detección en los tres depósitos donde los parámetros termohigrométricos, la naturaleza de los sustratos y las condiciones higiénicas difirieron.

Las Secciones Restricti y Aspergillus agrupan especies que se caracterizan por ser osmófílas, xerófilas y de lento crecimiento en condiciones de cultivo (Raper y Fennel, 1965; Klich y Pitt, 1994). Estas se desarrollan mejor a bajas actividades de agua (aw $\leq 0,8)$, incluso para muchas de ellas lo anterior es condición necesaria, tal es el caso de Aspergillus restrictus G. Smith, A. conicus Blochwitz y de algunos representantes del grupo $A s$ pergillus glaucus Link, detectados en este estudio (tabla II). A la hora de caracterizar la micobiota ambiental en ecosistemas secos, con altos niveles de salinidad o empolvamiento, si no se utilizan medios de cultivo que proporcionen cierto nivel tensión osmótica puede pasarse por alto la detección de especímenes de estos grupos importantes fundamentalmente por su impacto en la salud humana, el deterioro de alimentos en conserva y la afectación de materiales de interés patrimonial (De Hoog et al., 2000; Carrillo-Inungaray et al., 2006; Micheluz et al., 2015). Es recomendable en tales casos emplear medios de cultivo ricos como AM o Agar Dextrosa de Saboraud suplementados con $\mathrm{NaCl}(7,5$ a $15 \%)$ y extender el período de incubación hasta los 14 días. En MNBA se detectaron las mayores DR de Aspergillus glaucus y A. chevalieri Manguin (Sección Aspergillus) mientras de Restricti se detectó A.restrictus G. Smith y $A$. proliferans G.Smith. El otro depósito con buena representatividad de dichos grupos fue ARNAC. En ambos locales había una película consistente de polvo sobre el mobiliario y las piezas, a diferencia de OCPI donde los niveles de empolvamiento resultaron mucho menores. Esto pudiera explicar que en este último depósito se detectó solo una colonia de Aspergillus chevalieri (tabla II). En varios estudios de polvo casero se han detectado hongos del grupo Aspergillus glaucus y A. restrictus siendo frecuente la detección de $A$. chevalieri (Hicks et al., 2005; Nevalainen y Morawska, 2009; Visagie et al., 2014). Esta última especie, casi siempre en su fase holomórfica y otros representantes de las secciones en cuestión, se han reportado en el aire y sobre varios materiales en museos, archivos, bibliotecas y almacenes en varias regiones del mundo incluido Cuba (Sterflinger, 2010; Rojas y Aira, 2012). Micheluz et al. (2015) utilizando técnicas de microscopía electrónica y biología molecular demostraron abundante colonización en encuadernaciones y páginas de libros en una biblioteca de la Universidad $\mathrm{Ca}^{\prime}$ Foscari, Venecia (Italia) por hongos xerófilos del grupo Aspergillus glaucus y $A$. restrictus como $A$. halofilicus y $A$. proliferans que causaron daños importantes a los fondos. Aunque no en altas concentraciones especies de las secciones Nigri y Versicolor se aislaron en los depósitos (tabla II). Estos grupos constituyen contaminantes habituales en el interior de edificios con las características de los estudiados, sus propágulos pueden proceder del polvo o de la colonización de la superficies de diferentes materiales de origen orgánico como papel, yeso y cuero (Sterflinger, 2010; Harkawy et al., 2011).

\section{Biodeterioro de materiales}

Las potencialidades biodeteriorantes de todos los aislados fueron evaluadas in vitro (tabla III). El análisis general mostró a la excreción de ácidos orgánicos $(83 \%)$ y la actividad celulolítica sobre la carboximetilcelulosa (CMCasa) (86\%) como los fenómenos más evidenciados. Lo anterior es indicativo que los materiales de naturale- 
za celulósica tales como la madera el papel y los textiles almacenados en los depósitos constituyen un blanco importante como fuente de nutrientes para estos organismos. Son varios los ácidos orgánicos producidos por Aspergillus spp. (ácido cítrico, glucónico, palmítico, oxálico, esteárico, oleico y linoléico) (Dai et al., 2004). Estos hidrolizan el enlace $\beta$-1,4-glicosídico desintegrando las fibras de celulosa que brinda fortaleza mecánica a dichos materiales, además de aumentar la actividad de las celulasas fúngicas (Molina et al., 2014). Por otra parte, los ácidos fúngicos ejercen un efecto biocorrosivo sobre los materiales pétreos como el yeso y los morteros facilitando la colonización de esos sustratos por otros hongos (generalmente acidófilos) (Ljaljević y Vukojević, 2009). Resultaron los mejores productores de ácidos en este estudio $A s-$ pergillus tubingensis Mosseray, y A. flavipes (Bainier \& Sartory) Thom \& Church. Se ha señalado que el crecimiento en forma de microcolonias (tal y como crecen sobre materiales como el papel) favorece la producción de ácidos en especies de $A s$ pergillus como las de la sección Nigri (Krijgsheld et al., 2012). Con relación a la excreción de celulasas, fue notable además la actividad celulasa total (FPasa) del 73\% de las cepas capaces de crecer sobre papel de filtro, lo que implica la posibilidad de excreción al medio de endocelulasas, exocelulasas y $\beta$-glucosidasas, que pueden degradar de forma parcial o total la celulosa del papel. El buen crecimiento que mostraron los representantes de las secciones Nigri, Flavi y Versicolor además de las especies Aspergillus niveus Blochwitz, A. janus Raper \& Thom y A. fumigatus Fresen. en dicha condición, es muestra de su eficiente complejo enzimático celulolítico. Lakshimi et al. (2014) obtuvieron resultados similares en cepas de Aspergillus niger y $A$. flavus aisladas del suelo de varias regiones de la india, con actividades CMCasa mayores que otros hongos celulólíticos como Trichoderma y Chaetomium.

La degradación del almidón se evidenció en un $79 \%$ de las cepas mientras que un $74 \%$ secre- tó proteasas (gelatinasas) (tabla III). Las amilasas y proteasas se encuentran entre las enzimas más secretadas por Aspergillus. Ruth y Aguilar (2012) en estudios del secretoma de diferentes especies detectaron que dichas enzimas persisten en diferentes condiciones (medios sólidos, líquidos y de diferente composición) y durante varias fases del desarrollo (aparición de tubos germinales y conidiogénesis), lo cual sugiere un papel importante en el crecimiento fúngico. Rojas et al. (2009) evaluaron la actividad hidrolítica en hongos aislados de papel resultando la hidrólisis del almidón la actividad más común y las cepas de Aspergillus spp. las más activas. Las especies de la secciones Flavi, Usti y Versicolor se mostraron como los mejores productores de amilasas en este estudio, estas además de las ubicadas en la seccion Nigri, Aspergillus fumigatus y A. candidus Link. degradaron la gelatina. Ayansina y Owoseni (2010) reportaron alta secreción de amilasas en cepas de Aspergillus flavus aisladas de pan y demostraron que es directamente proporcional al aumento de la temperatura hasta valores cercanos a los $40^{\circ} \mathrm{C}$.

El almidón y la gelatina forman parte de los adhesivos utilizados para encuadernaciones de libros, pueden constituir parte importante del papel, el pergamino y de algunos textiles como los de algodón. Por otra parte, la gelatina constituye la emulsión de varias técnicas fotográfica (Vivar et al. 2013). Estos materiales en condiciones inadecuadas de T y HR se tornan altamente bioreceptivos por ende estos especímenes en los depósitos son una seria amenaza.

Aunque fue la actividad biodeteriorante menos detectada (34\%), la excreción de pigmentos debe tenerse en cuenta como un atributo importante por las afectaciones estéticas casi siempre irreversibles que pueden ocasionar en las colecciones (Borrego y García, 2011) (TablaIII). Se ha relacionado además, este fenómeno con la resistencia de determinadas especies a métodos de desinfección físicos y químicos (Bonifaz, 2012). En este aspecto fue notable la actividad que mostraron los repre- 
Tabla III. Resultados de las pruebas fisiológicas realizadas a las cepas para medir sus potencialidades biodeteriorantes.

\begin{tabular}{|c|c|c|c|c|c|c|c|c|}
\hline \multirow[b]{2}{*}{ Depósito } & \multirow[b]{2}{*}{$\begin{array}{l}\text { Identificación } \\
\text { taxonómica }\end{array}$} & \multicolumn{3}{|c|}{ Degradación de celulosa } & \multirow[b]{2}{*}{$\begin{array}{l}\text { Producción } \\
\text { de ácidos } \\
\text { (pH) }\end{array}$} & \multirow[b]{2}{*}{$\begin{array}{c}\text { Producción } \\
\text { de } \\
\text { pigmentos }\end{array}$} & \multirow{2}{*}{$\begin{array}{c}\begin{array}{c}\text { Actividad } \\
\text { amilolítica }\end{array} \\
\begin{array}{c}\text { Degradación } \\
\text { del almidón }\end{array}\end{array}$} & \multirow{2}{*}{$\begin{array}{c}\begin{array}{c}\text { Actividad } \\
\text { proteolítica }\end{array} \\
\begin{array}{c}\text { Liquefacción } \\
\text { de la gelatina }\end{array}\end{array}$} \\
\hline & & $\begin{array}{l}\text { Crecimiento } \\
\text { en papel de } \\
\text { filtro }\end{array}$ & $\begin{array}{l}\text { Crecimiento } \\
\text { en } \\
\text { carboximetil } \\
\text { celulosa }\end{array}$ & $\begin{array}{l}\text { Crecimiento } \\
\text { en celulosa } \\
\text { cristalina }\end{array}$ & & & & \\
\hline \multirow{9}{*}{ ARNAC } & A. clavatoflavus & + & + & + & 5.15 & - & + & + \\
\hline & A. conicus & - & $+/-$ & - & 6.57 & - & $+/-$ & - \\
\hline & A. glaucus 1 & - & $+/-$ & $+/-$ & 6.34 & + & + & + \\
\hline & A. glaucus 2 & - & - & - & 7.00 & - & - & - \\
\hline & A. niger & + & + & + & 4.08 & - & - & + \\
\hline & A. parasiticus & + & + & + & 5.87 & - & + & + \\
\hline & A. restrictus 1 & - & $+/-$ & - & 6.15 & - & $+/-$ & - \\
\hline & A. restrictus 2 & - & - & - & 7.00 & - & - & - \\
\hline & A. tubingensis & + & + & + & 3.70 & - & + & + \\
\hline \multirow{10}{*}{ OCPI } & A. candidus & $+/-$ & $+/-$ & $+/-$ & 6.07 & - & + & + \\
\hline & A. chevalieri & $+/-$ & - & - & 4.46 & - & + & + \\
\hline & A. flavipes & + & + & + & 3.72 & + & + & + \\
\hline & A. flavus & $H+$ & $H$ & H & 6.22 & - & + & + \\
\hline & A. niger & $+H$ & ++ & + & 5.41 & - & + & + \\
\hline & A. niveus & + & +4 & + & 5.30 & + & + & - \\
\hline & A. oryzae & + & + & + & 4.33 & + & + & + \\
\hline & A. unguis & + & + & + & 5.82 & + & + & + \\
\hline & A. versicolor & ++ & ++ & + & 4.13 & - & + & + \\
\hline & A. wentii & + & + & + & 4.82 & - & + & + \\
\hline \multirow{19}{*}{ MNBA } & A. chevalieri 1 & ++ & ++ & + & 4.32 & + & + & + \\
\hline & A. chevalieri 2 & $H$ & $H$ & + & 4.56 & + & + & + \\
\hline & A. conjunctus & + & + & + & 5.34 & - & + & + \\
\hline & A. flavipes & $+/-$ & + & $+/-$ & 3.87 & - & + & + \\
\hline & A. flavus & $+H$ & $+H$ & + & 6.03 & - & + & + \\
\hline & A. fumigatus & $H$ & $H+H$ & H & 4.10 & - & + & + \\
\hline & A. glaucus 3 & - & - & - & 7.00 & - & - & - \\
\hline & A. glaucus 4 & - & $+/-$ & $+/-$ & 6.54 & - & - & - \\
\hline & A. glaucus 5 & - & + & - & 6.48 & - & - & - \\
\hline & A. janus & + & + & + & 6.43 & + & + & + \\
\hline & A. niveus 1 & + & + & + & 6.43 & - & + & + \\
\hline & A. niveus 2 & $+H$ & + & + & 5.23 & - & + & + \\
\hline & A. ochraceous & + & + & + & 5.76 & + & + & + \\
\hline & A. proliferans & - & - & - & 5.65 & - & - & - \\
\hline & A. restrictus & - & $+/-$ & - & 6.23 & - & - & - \\
\hline & A. ustus 1 & $H+$ & $H+$ & +1 & 5.12 & + & + & + \\
\hline & A. ustus 2 & $H$ & + & + & 5.15 & + & + & + \\
\hline & A. ustus 3 & $+H$ & + & + & 5.76 & + & + & + \\
\hline & A. versicolor & + & + & + & 6.22 & + & + & + \\
\hline
\end{tabular}

H+: Indica crecimiento abundante, + : indica crecimiento moderado, + : indica crecimiento pobre, también es indicativo de la presencia de pigmento, degradación de almidóny degradación de gelatina. $+/-$ : indica crecimiento o producción de pigmento muy pobre, - : indica NO crecimiento, NO producción de pigmento y NO degradación de almidóny NO degradación de gelatina.

sentantes de las secciones Versicolor y Aspergillus además de Aspergillus unguis (Emile-Weill \& L. Gaudin) Thom \& Raper, A. ustus (Bainier) Thom $\&$ Church y $A$. ochraceus K. Wil. al excretar pigmentos con coloraciones de amarillo claro a pardo intenso.

Independientemente que un atributo biodeteriorante bajo determinadas condiciones sea suficiente para considerar un microorganismo dañino para determinado material (Borrego y García, 2011), la reunión de varios de ellos en un mismo espécimen sin duda alguna, le convierte en mucho más peligroso al ser capaz de infligir daños de diferente naturaleza sobre un mismo soporte o afectar diferentes materiales a la vez. Las especies de Aspergillus se encuentran entre los agentes mi- 
crobianos que desencadenan un amplio espectro de actividades biodeteriorantes sobre materiales de naturaleza orgánica (Molina et al, 2014). En el ARNAC el $45 \%$ de las cepas evidenciaron entre 4 y 5 de los atributos evaluados, mientras en MNBA lo hizo el 74\% (figura 2). Por ende en este último depósito son mucho mayores las posibilidades de afectaciones a la colección por las especies de $A s-$ pergillus.

\section{Potencial patogénico}

Aspergillus spp. es un patógeno oportunista que suele afectar personas con mecanismos de defensa comprometidos. Causa un grupo de síndromes clínicos de distribución universal conocidos como aspergilosis. Los tipos más frecuentes son: pulmonar, diseminada, cutánea, ótica, oftálmica y estados de hipersensibilidad inmunológica (alergias) (Alcalá et al. 1999; Bonifaz, 2012). Además del estado inmunitario del individuo, su ocupación desempeña una función específica en la aparición de este tipo de afecciones. El personal que labora en depósitos de archivos bibliotecas y museos puede estar expuesto a gran cantidad de conidios de Aspergillus, sobre todo por la tendencia de estos a adherirse a las partículas de polvo (Bonifaz, 2012). Algunas aspergilosis como las invasoras plantean para su diagnóstico certero un difícil reto al microbiólogo clínico (Palacio et al., 2003). La información cuantitativa y cualitativa acerca de la exposición del paciente a especies de Aspergillus en su entorno, pudiera tenerse en cuenta para el diagnóstico de la enfermedad y la determinación de su agente causal. Se relacionan con aspergilosis en humanos especies de 12 grupos, 9 de los cuales se detectaron en este estudio. Ellos son: Aspergillus fumigatus, A. flavus, A. niger, A. versicolor, A. nidulans, $A$. glaucus, $A$. candidus, A. flavipes y $A$. ustus (tabla II). En MNBA y OCPI se detectaron representantes de 6 de estos grupos mientras en ARNAC se detectaron especies que se ubican en tres de ellos. Visto así, los dos primeros depósitos pueden considerarse de mayor riesgo para la salud. Por otra parte, si se tienen en cuenta las $\mathrm{UFC}^{-3}$ por local (figura 1A), en MNBA el peligro era mucho mayor. Además, en dicho local se detectó Aspergillus fumigatus principal causante de las aspergilosis humanas (Oliveira y Caramalho, 2014).

Hay una serie de factores de virulencia asociados a la patogenicidad de cepas de Aspergillus, estos se despliegan y determinan el alcance y magnitud de las afecciones; la virulencia de este hongo como de otras especies funciona de forma multifactorial (Ortoneda et al., 2004; Bonifaz, 2012).
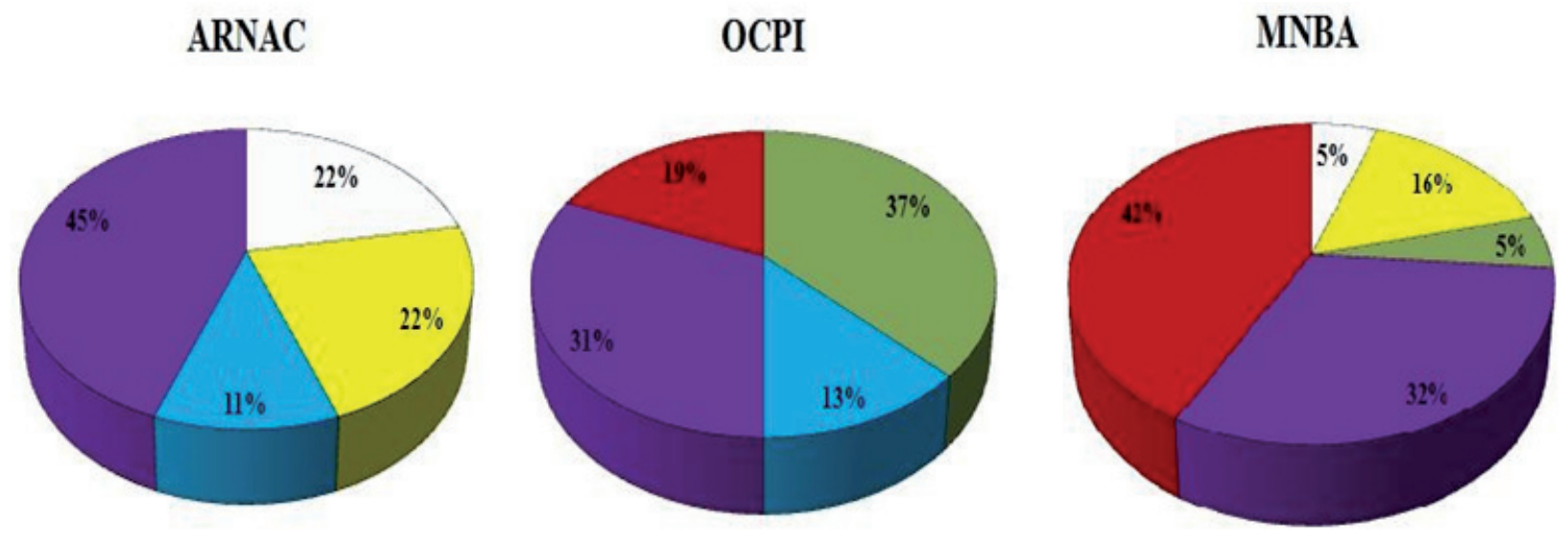

\section{Número de atributos: $\square$ cero $\square$ uno $\square$ dos $\square$ tres $\square$ cuatro $\square$ cinco}

Figura 2. Relación porcentual de las cepas detectadas en cada depósito según la cantidad de atributos biodeteriorantes que evidenciaron. 
La aspergillosis pulmonar se produce a partir de la inhalación de los conidios y su posterior desarrollo en el parénquima pulmonar. Es condición necesaria para ello que los conidios alcancen los sacos alveolares, donde pueden germinar, atravesar su pared y desencadenar un proceso invasivo (Bonifaz, 2012). El tracto respiratorio funciona como una compleja red cuyos conductos se van ramificando y disminuyendo su diámetro progresivamente hasta los alvéolos, solo los conidios de diámetro inferior a 3-4 $\mu \mathrm{m}$ llegarán hasta ese nivel (Hatch, 2009). Es por tanto, el pequeño tamaño de los conidios de Aspergillus spp. un importante factor de virulencia (tabla IV). El 47\% de los aislados en este estudio producen conidios que pueden ser arrastrados durante la respiración hasta los alveolos pulmonares. Especies como Aspergillus fumigatus, A. flavus y $A$. versicolor de notable capacidad invasiva en

Tabla IV. Resultados de las pruebas fisiológicas realizadas a las cepas para medir sus potencialidades patogénicas.

\begin{tabular}{|c|c|c|c|c|c|}
\hline Depósito & Cepa & $\begin{array}{c}\text { Tamaño conidial } \\
(\mu \mathrm{m})\end{array}$ & $\begin{array}{c}\text { Zona de } \\
\text { depositación en TR } \\
\end{array}$ & $\begin{array}{c}\text { Producción de } \\
\text { hemolisinas }\end{array}$ & $\begin{array}{c}\text { Crecimiento } \\
\text { a } 37^{0} \mathrm{C} \\
\end{array}$ \\
\hline \multirow{9}{*}{ ARNAC } & A. clavatoflavus & $3-3,5 \times 2,5-3$ & $\mathrm{~A}, \mathrm{~B}$ y C & + & $+\mathrm{a}$ \\
\hline & A. conicus & $4-4,5$ & A y B & - & - \\
\hline & A. glaucus 1 & $4,8-5,5$ & A y $B$ & - & $+/-$ \\
\hline & A. glaucus 2 & $4,5-5,3$ & A y $B$ & - & - \\
\hline & A. niger & $4-5$ & A y $B$ & + & $+\mathrm{a}$ \\
\hline & A. parasiticus & $3,5-5,5$ & A, B y C & + & $+\mathrm{a}$ \\
\hline & A. restrictus 1 & $4,5-5 \times 3-3,5$ & A y B & - & $+/-$ \\
\hline & A. restrictus 2 & $4,5-4,7 \times 3-3,5$ & A y $B$ & - & - \\
\hline & A. tubingensis & $3,2-4,3$ & $A, B$ y $C$ & + & $+\mathrm{a}$ \\
\hline \multirow{10}{*}{ OCPI } & A. candidus & $2,5-4$ & A, B y C & - & + \\
\hline & A. versicolor & $2-3$ & $A, B$ y $C$ & + & + \\
\hline & A. chevalieri & $4,4-5,3$ & A y B & + & + \\
\hline & A. flavipes & $2-3$ & A, B y C & - & + \\
\hline & A. flavus & $3,6-3,9$ & A, B y C & + & $+\mathrm{a}$ \\
\hline & A. niger & $4-5$ & A y B & + & $+\mathrm{a}$ \\
\hline & A. niveus & $2-2,5$ & A, B y C & + & $+\mathrm{a}$ \\
\hline & A. oryzae & $5-8 \times 4,5-7$ & A y $B$ & + & + \\
\hline & A. unguis & $2,5-3,5$ & $A, B$ y $C$ & - & - \\
\hline & A. wentii & $4,5-5$ & A y B & - & - \\
\hline \multirow{19}{*}{ MNBA } & A. chevalieri 1 & $4,4-5,3$ & A y B & - & + \\
\hline & A. chevalieri 2 & $4,6-5,1$ & A y $B$ & - & + \\
\hline & A. conjunctus & $3-3,5$ & $A, B$ y $C$ & + & + \\
\hline & A. flavipes & $2-3$ & $A, B$ y $C$ & + & + \\
\hline & A. flavus & $3,6-3,9$ & $A, B$ y $C$ & + & $+\mathrm{a}$ \\
\hline & A. fumigatus & $2,5-3$ & $A, B$ y $C$ & + & $+\mathrm{a}$ \\
\hline & A. glaucus 3 & $4,8-5,5$ & A y $B$ & - & - \\
\hline & A. glaucus 4 & $4,5-5,3$ & A y $B$ & + & $+/-$ \\
\hline & A. glaucus 5 & $4,5-5,3$ & A y $B$ & - & - \\
\hline & A.janus & $2-2,5$ & $A, B$ y $C$ & + & + \\
\hline & A. niveus 1 & $2-2,5$ & $A, B$ y $C$ & - & + \\
\hline & A. niveus 2 & $2-2,5$ & $A, B$ y $C$ & + & + \\
\hline & A. ochraceous & $2,5-3,5$ & A, B y C & + & - \\
\hline & A. proliferans & $3,5-7,2$ & A y B & - & $+/-$ \\
\hline & A. restrictus & $4,5-5 \times 3-3,5$ & A y $B$ & - & - \\
\hline & A. ustus 1 & $3-4,5$ & A y $B$ & + & + \\
\hline & A. ustus 2 & $3-4,5$ & A y B & + & + \\
\hline & A. ustus 3 & $3-4,5$ & A y $B$ & + & + \\
\hline & A. versicolor & $2-3$ & A, B y C & + & + \\
\hline
\end{tabular}

+: Indica Producción de hemolisinas y crecimiento a $37^{\circ} \mathrm{C} .+/-$ : Indica poco crecimiento a $37^{\circ} \mathrm{C}$. ${ }^{\mathrm{a}}:$ Indica crecimiento abundante a $37^{\circ} \mathrm{C}$. TR: Tracto

Respiratorio. Se deposita en: A: TR superior, B: Tráquea, Bronquiosy Bronquiolos, C: Alveolos pulmonares. 
hospederos humanos susceptibles presentaron esta característica. Entre los principales atributos patogénicos de la mayoría de las cepas se encuentra su capacidad de crecer a $37^{\circ} \mathrm{C}$, temperatura corporal del hombre y otros animales homeotermos. El desarrollo bajo dicha condición posibilita la invasión de superficies epiteliales, endoteliales y vasos sanguíneos (Alcalá et al. 1999). Por otra parte, a dicha temperatura se induce la activación de varios genes implicados en mecanismos invasivos (Rementería et al., 2005; Mccormick et al., 2010). Un 66\% de los aislados de forma general, crecieron bajo dicha condición a la cual se inhibe el desarrollo de muchos hongos saprobios (Llop et al., 2001). Destacaron al crecer de forma exhuberante Aspergillus fumigatus, A. niveus Blochwitz y los aislados ubicados en las secciones Nigri y Flavi (tabla IV). Estas especies pueden desarrollarse en un amplio rango de temperaturas, en algunos casos sus temperaturas óptimas de crecimiento incluyen la corporal. Tales son los casos de Aspergillus niger (35$\left.37^{\circ} \mathrm{C}\right)$, A. oryzae $\left(30-37^{\circ} \mathrm{C}\right)$ y A. fumigatus $\left(37^{\circ} \mathrm{C}\right)$ (Krijgsheld et al., 2012). En casos de Aspergiloma y Aspergilosis invasora se han reportado como los principales agentes causales Aspergillus fumigatus, A. flavus, A. niger cuestión condicionada en gran parte por su potencial de desarrollo a $37^{\circ} \mathrm{C}$.

Las hemolisinas han sido clásicamente definidas como exotoxinas capaces de lisar eritrocitos y otras células nucleadas. Se ha sugerido que interactúan con ligandos específicos sobre la superficie de varios blancos celulares formando poros que conllevan a la lisis celular producto de desbalance osmótico. Las hemolisinas fúngicas han sido propuestas como importantes factores de virulencia (Rementeria et al., 2005; Bonifaz, 2012; Nayak et al., 2013). Con la lisis de los eritrocitos se libera hierro, un importante factor de crecimiento microbiano en especial durante las infecciones (Bullen, 1981). Se ha sugerido que estas micotoxinas proveen una estrategia de supervivencia durante las infecciones oportunistas por sus efectos citotóxi- cos sobre otros tipos celulares como leucocitos y células nerviosas (Nayak et al., 2011). Algunos investigadores señalaron que las hemolisinas pueden jugar un rol importante en afectaciones a la salud producto del llamado Síndrome del Edificio Enfermo (Vesper y Vesper, 2004). Fueron hemolíticos en este estudio el $58 \%$ de los aislados, entre ellos los correspondientes a las Secciones Flavi, Nigri, Versicolor y Usti además de especies como Aspergillus fumigatus y A. ochraceus (tabla IV). Nayak et al. (2013) reportaron la caracterización de más de un tipo de hemolisina en cepas de $A$. fumigatus, A. flavus, A. oryzae y A. niger, especies productoras por excelencia de este tipo de micotoxinas y de gran importancia clínica. En ensayos "in vivo" de ratones inoculados con esporas de A. fumigatus se han detectado estas micotoxinas en el suero; se han comprobado daños en pulmones, hígado, cerebro, corazón, riñones así como un aumento en la capilaridad de los vasos sanguíneos (Berne et al., 2009; Chaudhary et al., 2012).

Aspergillus glaucus, A. chevalieri y A. niveus mostraron diferencias entre cepas para la actividad hemolítica, cuestión que evidencia que dicho atributo patogénico, como muchos otros puede diferir entre variedades y cepas de una misma especie, incluso en aislados de ecosistemas similares (Molina y Borrego, 2014).

Conjuntamente con la magnitud en la que se manifiesta un factor de virulencia, la reunión en un mismo microorganismos de varios de ellos es condición necesaria para considerarlo potencialmente patógeno (Molina et al., 2014). En el ARNAC el $33 \%$ de las cepas crecieron a $37^{\circ} \mathrm{C}$, secretaron hemolisinas, y su tamaño conidial posibilita la depositación en alvéolos. En OCPI y MNBA lo hicieron el 30 y 37\% respectivamente (figura $3)$. Se evidenció que en el último local existía mayor peligro de afecciones a la salud por Aspergillus spp., si se tienen en cuenta además los aspectos ecológicos, microclimáticos y sanitarios analizados con anterioridad. 

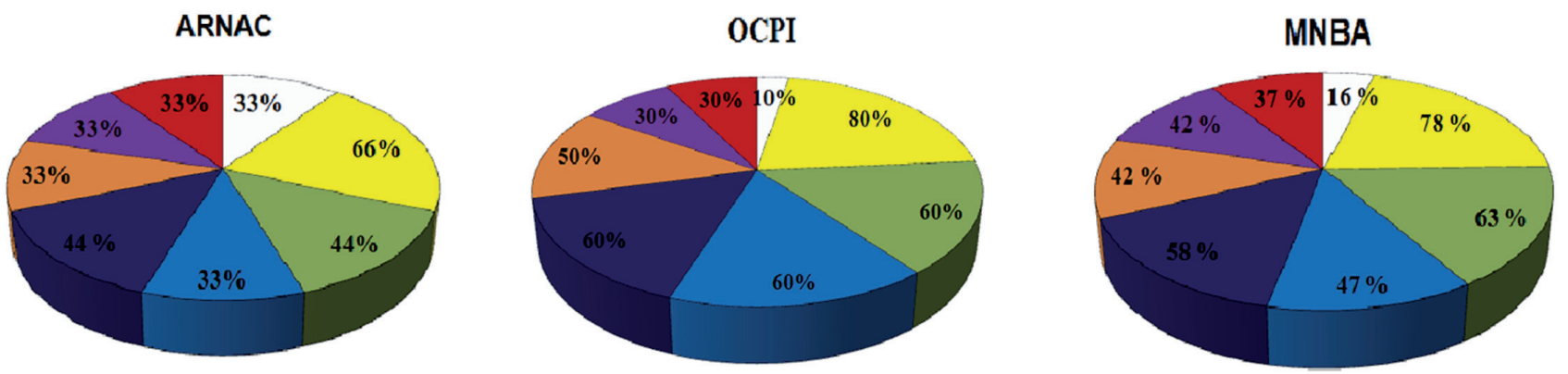

$$
\begin{array}{ll}
\square \text { Ninguno } & \square \text { Crecimiento a } 37^{\circ} \mathrm{C}\left(37^{\circ} \mathrm{C}\right) \\
\square \text { Secreción de hemolisinas (HL) } & \square \text { Deposición en alveolos (AL) } \\
\square 37^{\circ} \mathrm{C} \times \mathrm{HL} & \square 37^{\circ} \mathrm{C} \times \mathrm{AL} \\
\square \mathrm{HL} \times \mathrm{AL} & \square 37^{\circ} \mathrm{C} \times \mathrm{HL} \times \mathrm{AL}
\end{array}
$$

Figura 3. Relación porcentual de las cepas detectadas en cada depósito según los atributos patogénicos que evidenciaron.

\section{CONCLUSIONES}

Aspergillus spp. constituyó una parte importante de la micobiota aérea viable en los depósitos estudiados. MNBA fue el local con mayor prevalencia del género en términos de concentración y variabilidad. Su presencia en el ecosistema aéreo estuvo correlacionada de forma positiva con la HR y guardó relación con la ventilación y la presencia de polvo. Se detectaron 22 especies correspondientes a 12 secciones diferentes, las secciones de mayor variabilidad y representatividad fueron Flavi, Restricti y Aspergillus. Entre los aislados se detectaron especies y grupos de amplio potencial adaptativo, además de otros (como Aspergillus glaucus y $A$. restrictus) que encuentran en este tipo de ambientes las condiciones favorables para su desarrollo. Las potencialidades biodeteriorantes de los aislados evidenciaron la capacidad de la mayoría para provocar daños significativos a los materiales, en especial los de naturaleza orgánica. Varias cepas correspondieron a especies patógenas oportunistas, crecieron bien a temperatura corporal y fueron hemolíticas. La permanencia de personal desprotegido o inmunodeprimido en estos depósitos puede propiciar la aparición de diferentes clases de aspergilosis. Los aislados con mayor impacto biodeteriorante y patogénico fueron los correspondientes a las Secciones Flavi, Nigri y la especie Aspergillus fumigatus.

\section{REFERENCIAS}

Abrusci, C., González, A., Del Amo, A., Catalina, F., Collado, J., Platas, G. (2005). Isolation and identification of bacteria and fungi from cinematographic films. Internacional Biodeterioration and Biodegradation. 5: 58-68.

Alcalá, L., Muñoz, P., Peláez, T., Bouza, E. (1999). Aspergillus y aspergilosis. Servicio de Microbiología Clínica. Hospital General Universitario Gregorio Marañón, España. Control Calidad SEIMS.
Almaguer, M. y Rojas, T.I. (2013). Aeromicota viable en la atmósfera de La Habana, Cuba. Nova Acta Científica Compostelana (Bioloxía). 20: 35-45.

Anaya, M., Borrego, S., Cobo, H., Valdés, O., Molina, A. (2014). Aeromicobiota de un depósito de alimentos en La Habana, Cuba. Augmdomus. 6: 95-110.

Ayansina, A. D. V., Owoseni, A.A. (2010). Studies on Amylolytic enzyme Synthesized by Aspergillus 
flavus Associated with Mouldy Bread. Pakistan Journal of Nutrition. 9 (5): 434-437.

Barnett, H.L. y Hunter, B.B. (2003). Illustrated genera of Imperfect fungi. 4th ed. Burgués. Publishing Company Minneapolis 15, Minn.

Berne S., Lah, L., S, K. (2009). Aegerolysins: structure, function, and putative biological role. Protein Sci 18:694 - 706.

Bipasha, G., Lal , H., Srivastava, A. (2015). Review of bioaresols in indoor environment with special reference to sampling, analysis and control mechanisms. Environment International. 85: 254-272.

Bogomolova, E.V., Kirtsideli, I. (2009). Airborne fungi in four stations of the St. Petersburg underground railway system. International Biodeterioration and Biodegradation. 63(2):156-160.

Bonifaz, A. (2012). Micología Médica Básica. Editorial Interamericana Mc Grant Hill. Cuarta Edición. 570 p.

Borrego, S. y García, M. (2011). Comportamiento de la concentración microbiana aérea en la Fototeca del Archivo Nacional de Cuba. Revista CENIC Ciencias Biológicas. 42: 61-67.

Borrego, S. y Molina, A. (2014). Comportamiento de la aeromicrobiota en dos depósitos del Archivo Nacional de la República de Cuba durante 7 años de estudio. AUMGMDOMUS. 6:1-24.

Borrego, S., Guiamet, P., Gómez de Saravia, S., Battistoni, P., García, M., Lavín, P., Perdomo, I. (2010). The quality of air at archives and the biodeterioration of photographs. International Biodeterioration and Biodegradation. 64: 139-145.

Bučková, M., Puškárová, A., Sclocchi, M.C., Bicchieri, M., Colaizzi, P., Pinzari, F., Pangallo, D. (2014). Co-occurrence of bacteria and fungi and spatial partitioning during photographic materials biode- terioration. Polymer Degradation and Stability. 108: $1-11$.

Bullen, JJ. (1981). The significance of iron in infection. Rev Infect Dis. 3:1127-1138.

Cabral, P.S.J. (2010). Can we use indoor fungi as bioindicators of indoor of indoor air quality? Historical perspectives and open questions. Science of the total environment. 408: 4285-4295.

Carrillo-Inungaray, M.L., Ramírez-Zapata, M.R., Martinez-Castilleja, J.C. (2006). Efecto de solutos sobre el crecimiento de hongos deteriorativos de alimentos. Ciencia y Tecnología Alimentaria. 5(2): 142-146.

Casadesús, L.y Rojas, T.I. (1981). Micología. Manual Práctico. Ed. MES; La Habana. 99 pp.

Chaudhary, N., Datta, K., Askin, F.B., Staab, J.F., Marr, K.A. (2012). Cystic fibrosis transmembrane conductance regulator regulates epithelial cell response to Aspergillus and resultant pulmonary inflammation. American journal of respiratory and critical care medicine. 185(3): 301-10.

Codina, R., Fox, R.W., Lockey, R.F., DeMarco, P., Bagg, A. (2008). Typical Levels of Airborne Fungal Spores in Houses Without Obvius Moisture Problems During a Rainy Season in Florida, Usa. J Investig Allergol Clin inmunol. 18(3): 156-162.

Dai, Z., Mao, X., Magnuson, J., Lasure, L. (2004). Identification of genes associated with morfhology in Aspergillus niger by using suppression subtractive hybridization. Applied and Environmmental Microbiology. 70: 2474-2485.

De Hoog, G.S., Guarro, G., Gene, J. Figueras, M.J. (2000). Atlas of clinical fungi. 2nd ed. Universidad Rovira I Virgili Reus, España. 1126 pp.

Gaikwad, K.N y Sonawane M.D. (2012). Fungi as bio-indicators of air quality. International Journal of Life science \& Pharma Research. 2: 25-28. 
Guiamet, P.S, Borrego, S., Lavin, P., Perdomo, I., Gómez de Saravia, S. (2011). Biofouling and biodeterioration in material stored at the Historical Archive of the Museum of La Plata, Argentine and at the National Archive of the Republic of Cuba. Colloids and Surfaces B: Biointerfaces. 85(2): 229-234.

Harkawy, A., Górny, R. L., Ogierman, L., Wlazlo, A., Lawniczek- Wałczyk, A., Niesler, A. (2011). Bioaerosol assessment in naturaly ventilated historical library building with restricted personnel access. Annals of Agricultural and Environmental Medicine. 18(2) 323-329.

Hatch, T.F. (2009). Distribution and deposition of inhaled particles in Respiratory Tract. Mmbr. Asm. Org. 25: 237-240.

Hicks, J.B., Lu, E.T., De Guzman, R., Weingart M. (2005) Fungal types and Concentrations from Settled Dust in Normal Residences. Journal of Occupational and Environmental Hygiene. 2(10): 481-492.

Horner, E., Worthan, A. G., Morey, F.R. (2004). Air- and dustborne Mycoflora in houses Free of Water Damage and Fungal Growth. Applied and environmental Microbiology. Nov. 2004: 6394-6400.

Klich, M.A. y Pitt, J.I. (1994). A Laboratory guide to the common Aspergillus species and their teleomorphs. Commonwealth Scientific and Industrial Research Organization. 116 p.

Krijgsheld, P., Bleichrodt, R., van Veluw, G.J., Wang, F., Müller, W.H., Dijksterhuis, J., Wösten, H.A.B. (2012). Development in Aspergillus. Studies in Mycology. 74: 1-29.

Lakshimi, N. R., Surech, B., Radhaiah, A., Sreeramulu, A. (2014). Screening, Identification and Isolation of Cellulolytic fungi from soils of Chittoor District, India. International Journal of Current Microbiology and Applied Sciences. 3(7): 761-771.
Lavin, P., Gómez de Saravia, S.G., Guiamet, P.S. (2014). An environmental assessment of biodeterioration in document repositories. Biofouling. 30(5): 561-569.

Ljaljević, M.V. y Vukojević, J.B. (2009). Role of fungi in biodeterioration process of stone in historic buildings. Zbornik Matice Srpske za Prirodne Nauke. 116: 245-251.

Llop, A., Váldez-Dapena, M., Zuazo, J. (2001). Microbiología y Parasitología médica. Editorial Ciencias Médicas, La Habana, 550 pp.

McCormick, A., Loeffler, J., Ebel, F. (2010). Aspergillus fumigatus: contours of an opportunistic human pathogen. Cellular Microbiology. 12(11):1535-1543.

Micheluz, A., Manente, S., Tigini, V., Prigione, V., Pinzari, F., Ravagnan, G., Varese, G. (2015). The extreme environment of a library: Xerophilic fungi inhabiting indoor niches. International Biodeterioration and Biodegradation. 99:1-7.

Molina, A. y Borrego, S. (2014). Análisis de la micobiota existente en el ambiente interior de la mapoteca del Archivo Nacional de la República de Cuba. Boletín Micológico. 29(1): 2-17.

Molina, A. y Borrego, S. (2014). Caracterización de hongos aislados de mapas conservados en el Archivo Nacional de la República de Cuba. Ge-conservación. 6: 35-44.

Molina, A., Valdés, O., Borrego, S., Pérez, D., Castro, M. (2014). Diagnóstico micológico ambiental en depósitos de la Oficina Cubana de la Propiedad Industrial. Nova Acta Científica Compostelana (Bioloxía). 21:107-117.

Nayak, A., Green, B. y Beezhold, D. (2013). Fungal hemolysins. Medical Mycology. 51:1-16. 
Nayak, A.P., Blachere, M., Hettick, J.M., Lukomski, J.M.S., Schmechel, S., Beezhold, D.H. (2011). Characterization of recombinant Terrelysin, a hemolysin of Aspergillus terreus. Mycopathologia.171:23-34.

Nevalainen, A. y Morawska, L. (eds). (2009). Biological Agents in Indoor Environments. Assessment of Health Risks. Work conducted by a WHO Expert Group between 2000-2003. [en línea] http://www.ilaqh.qut.edu.au/Misc/BIOLOGICAL_ AGENTS_2009.pdf 03/09/2015.

Oliveira, M. y Caramalho, R. (2014). Aspergillus fumigatus: a mere bioaerosol or a powerful biohazard? Nova Acta Científica Compostelana (Bioloxía). 21: 57 - 64.

Ortoneda, M., Guarro, J., Madrid, M.P., Caracuel, Z., Roncero, M.I., Mayayo, E. y Di Pietro, A. (2004). Fusarium oxysporum as a Multihost Model for the Genetic Dissection of Fungal virulence in Plants and Mammals. Infection and Inmunity .72: 1760-1776.

Palacio, A., Cuétara, M.S., Pontón, J. (2003). El diagnóstico de laboratorio de la aspergilosis invasora. Revista Iberoamericana de Micología. 20: 90-98.

Piontelli, E. (2008). Aportes morfotaxonómicos en el género Aspergillus Link: Claves para las especies ambientales y clínicas más comunes. Boletín Micológico. 23: 49-66.

Raper, K.B. y Fennel, D.I. (1965): The Genus Aspergillus. The Williams y Wilkins Co. Baltimore, USA. 689 p.

Rementeria, A., López-Molina, N., Ludwig, A. (2005). Genes and molecules involved in Aspergillus fumigatus virulence. Revista Iberoamericana de Micología. 22: 1-2.

Resolución No. 41 (2009). Lineamientos para la conservación de las fuentes documentales. Ministerio de Ciencia Tecnología y Medio Ambiente (CIT-
MA), Gaceta oficial 9 de mayo 2009. [en línea] http://www.gacetaoficial.cu/pp.193

Rodríguez, J.C., Rodríguez, B., Borrego, S. F. (2014). Evaluación de la calidad micológica ambiental del depósito de fondos documentales del Museo Nacional de la Música de Cuba en época de lluvia. 6: 123-146.

Rojas, J.A., Cruz, C., Mikán, J.F., Villalba, L.S., Cepero de García, M.C., Restrepo, S. (2009). Isoenzyme characterization of proteases and amylases and partial purification of proteases from filamentous fungi causingbiodeterioration of industrial paper. International Biodeterioration \& Biodegradation 63 (2009) 169-175.

Rojas, T y Aira, M.J. (2012). Fungal biodiversity in Indoor Environments in Havana, Cuba. Aerobiologia. 28: 367-374.

Rojas, T.I., Llanes, N., Benitez, M., Aira, M. J., Malagón, H. (2007). El género Aspergillus en la atmosfera de la Habana (Cuba). Boletín Micológico. 22: 41- 46 .

Rojas, T.I., Martínez, E., Aira, M.J., Almaguer, M. (2008). Aeromicota de ambientes internos: Comparación de métodos de muestreo. Boletín Micológico. 23: 67-73.

Ruth, L.T. y Aguilar G.O. (2012). El Estado actual del proteoma de Aspergillus. BioTecnología. 16 (2): 129-151.

Samson, R.A., Visagie, C.M., Houbraken, J., Hong, S.B., Hubka, V., Klaassen, C.H.W., Perrone, G., Seifert, K.A., Susca, A., Tanney, J.B.,Varga, J., Kocsubé, S., Szigeti, G., Yaguchi, T., Frisvad, J.C. (2014). Phylogeny, identification and nomenclature of the genus Aspergillus. Studies in Mycology. 78: 141-173.

Sánchis, J. (2002). Los nueve parámetros más críticos en el muestreo microbiológico del aire. Revis- 
ta Técnicas de Laboratorio. 276: 858- 862.

Sepúlveda, C. y Piontelli, E. (2005). Poblaciones de Aspergillus en semillas de maíz y soja de importación Argentina: Énfasis en la sección Flavi. 20: 41-55.

Simões, M. F., Santos, C., Lima, N. (2013). Structural Diversity of Aspergillus (Section Nigri) Spores. Microscopy and Microanálisis. 19 (5): 1151- 1158.

Smith, G. (1980). Ecology and Field Biology. 2nd Edition. Harper \& Row (eds.), New York.

Sterflinger, K. (2010). Fungy: Their role in deterioration of culturable heritage. Fungal Biology Reviews. 24: 47-55.
Vesper, S.J. y Vesper, M.J. (2004). Possible roles of fungal hemolysins sick building syndrome. Advances in Applied Microbiology.55:191-213.

\section{Visagie, C.M., Hirooka, Y., Tanney, J.B., Mwan-} ge, K., Meijer, M., Amend, A.S., Seifert, K.A., Samsom, R.A. (2014). Aspergillus, Penicillium and Talaromyces isolated from house dust samples collected around the word. Studies in Mycology. 78: 63-139.

Vivar, I., Borrego, S., Ellis, G., Moreno, D.A., García, A. M. (2013). Fungal biodeterioration of color cinematographic films of the cultural heritage of Cuba. International Biodeterioration and Biodegradation. 84: 372-380. 